\section{Case Reports in Ophthalmology}

\title{
Endoscope-Assisted and Controlled Argus II Epiretinal Prosthesis Implantation in Late-Stage Retinitis Pigmentosa: A Report of 2 Cases
}

\author{
Emin Özmert Sibel Demirel \\ Ankara University Faculty of Medicine Department of Ophthalmology, Ankara, Turkey
}

\section{Keywords}

Argus II · Retinal prosthesis · Artificial vision - Retinal tack · Array · Endoscope

\begin{abstract}
Several different approaches for restoring sight in subjects who are blind due to outer retinal degeneration are currently under investigation, including stem cell therapy, gene therapy, and visual prostheses. Although many different types of visual prostheses have shown promise, to date, the Argus II Epiretinal Prosthesis System, developed in a clinical setting over the course of 10 years, is the world's first and only retinal prosthesis that has been approved by the United States Food and Drug Administration (FDA) and has been given the CE-Mark for sale within the European Economic Area (EEA). The incidence of serious adverse events from Argus II implantation decreased over time after minor changes in the implant design and improvements in the surgical steps used for the procedure had been made. In order to further decrease the scleral incision-related complications and enhance the assessment of the tack position and the contact between the array and the inner macular surface, we used an ophthalmic endoscope during the regular course of Argus II implantation surgery in 2 patients with late-stage retinitis pigmentosa in an attempt to improve the anatomical and functional outcomes.




\section{Case Reports in Ophthalmology}

\section{Introduction}

Outer retinal degenerative diseases such as retinitis pigmentosa (RP) and dry agerelated macular degeneration destroy photoreceptors but leave a significant percentage of the inner retinal cells (ganglion and bipolar cells) intact and functional [1-3]. Several different approaches for restoring sight in subjects who are blind due to outer retinal degeneration are currently under investigation, including stem cell therapy, gene therapy, and visual prostheses. On this basis, vision-restoring implants have been developed to interface with various parts of the visual pathway, particularly the retina [4]. One such device is the epiretinal implant, which targets retinal ganglion cells by having the electrodes contact the inner surface of the retina [3]. Although many different types of visual prostheses have shown promise, to date, the Argus II Epiretinal Prosthesis System (Second Sight Medical Products, Sylmar, CA, USA), which was developed in a clinical setting over a period of 10 years, is currently the world's first and only United States Food and Drug Administration (FDA) and CE-Mark-approved retinal prosthesis for subjects blinded by outer retinal degeneration. The Argus II Retinal Prosthesis System consists of a camera worn on a pair of glasses that transmits electrical signals to an array of 60 electrodes that are implanted on a patient's macula. The array bypasses the photoreceptor layer and directly stimulates the remaining inner retinal cells with a signal transmitted via the normal visual pathway to the visual cortex $[3,5,6]$.

Electrical stimulation to the remaining cells of the retina via the Argus II device produces spots of light, called phosphenes, which are visible to users of the device. Users learn to interpret these visual percepts, thereby enabling patients who are blind due to late-stage RP to regain a limited sense of sight $[2,3,5,7]$. The Functional Low-Vision Observer Rated Assessment (FLORA) was developed to evaluate functional visual ability and well-being in a population of subjects whose ultra-low vision has been restored by the Argus II Retinal Prosthesis System. Polling of the more than 100 RP patients who have been implanted with the Argus II indicates that navigation and orientation are among the major benefits of the device. It has also proven to be safe and stable during chronic implantation [8, 9]. A report by Second Sight Medical Products published in December 2015 states that Argus II can reliably withstand a long-term implant (>8 years) in a significant number of subjects with an acceptable safety profile. Using the system, blind subjects showed improved performance on visual tasks, and the results were sustained up to 5 years [10].

A transscleral cable connection is a weak point in all systems because it theoretically allows microorganisms to enter the eye, resulting in endophthalmitis. Leakage through the pars plana incision site may also cause serious postoperative hypotony. The incidence of serious adverse events (SAEs) decreased over time after minor changes had been made to the implant design of the Argus II system and after the surgical steps had been improved [4, 11]. A recent report noted that despite their low, but still significant, rate in all patients with commercial implants, SAEs still occurred. These were infectious endophthalmitis $(0.0 \%)$, hypotony $(3.4 \%)$, retinal retacking $(0.0 \%)$, retinal detachment $(3.4 \%)$, retinal break $(0.0 \%)$, sclerotomy leak (1.1\%), and explant (1.1\%) [10].

In an attempt to manage all these scleral incision-related complications in order to control the positions of retinal tack and the contact between the array and the inner surface of the macula, we used an ophthalmic microendoscope during the regular course of Argus II implantation surgery, which was proposed by Second Sight's Surgeon Manual in 2, late-stage RP patients [12]. To the best of our knowledge, this is the first study in the literature to report the use of this technique for late-stage RP patients. 


\section{Case Reports in Ophthalmology}

\section{Surgical Method}

The Argus II device consists of an extraocular component and an intraocular component, but no extra-orbital components. Therefore, the surgical approach is a complex combination of extraocular and intraocular procedures. The manufacturer calculates and prescribes the distances from the limbus for the scleral incision and the tab sutures based on the biometric data obtained from an ultrasound of the axial length prior to surgery. The surgical approach for epiretinal Argus II implantation uses standard vitreoretinal techniques without the routine use of silicone oil. Since aphakic or pseudophakic patients are preferable, lens removal 2 weeks before the implantation surgery or a pars plana lensectomy during the surgery is performed. Vitrectomy ports are prepared at the 3- to 9-o'clock positions, the infusion line is inserted at the inferotemporal quadrant, and 25-G chandelier light is introduced at the 6-o'clock position, which provides sufficient illumination during the bimanual work.

After placement of the extrascleral part, which consists of a receiving coil and an electronics case, a full vitrectomy following complete posterior vitreous detachment is performed with the assistance of triamcinolone acetonide to ensure that all vitreous remnants are removed from the vitreous base and the retinal surface. The standard concentration of this preparation is $40 \mathrm{mg} / \mathrm{mL}$. The triamcinolone is diluted with a balanced salt solution at about 1:4 before instilling in the eye during vitrectomy. A perpendicular 5.2-mm scleral incision through the pars plana has to be made in order to insert the cable, which has an epiretinal stimulator array of 60 microelectrodes at its tip. Just before creating the scleral incision, the prescribed setback distance from the limbus, which depends on the patient's axial length, is measured along the cable axis and marked at the center of the sclerotomy site (Fig. 1). A 30-G fine needle is inserted from the prepared central mark through the pars plana (Fig. 2). Using an endoscope, we decided to check the prescribed scleral incision site internally in order to confirm the proper location (fine-needle test). The 23-G nasal scleral incision, which is used in regular vitrectomy, was used to insert the 23-G fiberoptic-type endoscope probe with a 120-degree visual field (Model E2 MicroProbe, Laser and Endoscopy System, OME 2000; Endo Optiks, Inc., Little Silver, NJ, USA). With direct endoscopic viewing, we can control the pars plana, the trace of the fine needle, and the ora serrata (Fig. 3), which is impossible to do with the anteroposterior view of an operating microscope without a scleral indentation. If the scleral incision site, which is controlled internally by an endoscope, seems to be appropriate, a 5.2-mm-wide scleral incision is created to insert the array according to the manufacturer's instructions (Fig. 4). If the scleral incision site is unsuitable, the measurement to the limbus prescribed and provided by the manufacturer is revised. After making a scleral incision and inserting the electronics array, the retroiridal space is checked using an endoscope to determine any complications, such as damage to the ciliary body, choroidal detachment, or retinal tears (Fig. 5). If no scleral incision-related issues are observed, a surgeon can proceed to the surgical steps.

If everything is planned and performed according to the measurements and to the manufacturer's recommendations, the electrode array should land without any force at the macular area, and, ideally, it should not cover the optic disc. It is essential to position the device over the central macula with minimal space between the array and the retinal surface. If the stimulator is not centered in the macula, the narrowing of the sutures at the incision site must be modified. If everything looks appropriate, the implant is fixated using a retinal tack. The application of the retinal tack, its insertion, the release of the handle, and ensuring that the array makes good contact with the macular surface are the most critical parts of the procedure. We can check the position of the tack and the contact between the macular surface 
Case Reports in
Ophthalmology

Case Rep Ophthalmol 2016;7:315-324

DOI: $10.1159 / 000453606$

C 2016 The Author(s). Published by S. Karger AG, Basel www.karger.com/cop

Özmert and Demirel: Endoscope-Assisted and Controlled Argus II Epiretinal Prosthesis Implantation in Late-Stage Retinitis Pigmentosa: A Report of 2 Cases

and the array using the excellent side-viewing capability of the curved-probe of the endoscope, which is more effective than using the anteroposterior viewing of an operating microscope (Fig. 6). Nonperpendicular or shallow tacking causes the device to move away from the retina. At the end of the surgery, we have to control the peripheral retina and vitreous base without scleral indentation since the electronics were secured to the sclera at the beginning of the surgical procedure. All these steps were easily accomplished using an ophthalmic microendoscope with no endoscope-related complications without any need of scleral indentation.

\section{Cases}

This paper reports on 2 cases of patients with late-stage RP in whom an ophthalmic endoscope was used during the regular course of Argus II implantation surgery in order to improve the anatomical and functional outcomes.

Case 1

Case 1 was a 46-year-old male who has had late-stage RP for the past 10 years with minimal light perception and projection in both eyes. After the routine ocular examination, including spectral-domain optical coherence tomography and fundus autofluorescence, and a discussion about surgery, fitting/rehabilitation procedures, and the possible benefits and complications of the method, the subject underwent implantation of the Argus II Retinal Prosthesis System in his left eye, typically the worse-seeing eye, on December 28, 2015. An endoscope-assisted and controlled regular surgical procedure for Argus II epiretinal implantation was performed without any complications. The lens was removed just before the vitrectomy phase by pars plana lensectomy. The patient is currently undergoing his fourth rehabilitation session, and he can detect white plates and glasses on a dark table as well as some squares and lines on a screen.

\section{Case 2}

Case 2 involved a 31-year-old male who has had late-stage RP for the past 8 years with minimal light perception and projection in both eyes. After the routine ocular examination, including spectral-domain optical coherence tomography and fundus autofluorescence, and a discussion about the surgery, fitting/procedures, and the possible benefits and complications of the method, the patient underwent implantation of the Argus II Retinal Prosthesis System in his left eye, typically the worse-seeing eye, on June 27, 2016. Two weeks before the implantation surgery, phacoemulsification without intraocular lens implantation was performed. An endoscope-assisted and controlled regular surgical procedure was performed without any complications. He is currently undergoing his first rehabilitation session, and he can follow patterns of light on the floor.

In both cases, during the fitting procedure, we used complete active electrodes with the proper impedance measurement and perfect placement of the arrays. So far, there have not been any complications, such as a retinal tear, hypotony, ciliary body detachment, or tacking issues. 


\section{Case Reports in Ophthalmology}

\section{Discussion}

During the regular course of Argus II implantation surgery, we decided to use the 23-G fiber-fused type ophthalmic microendoscope due to its potential benefits and advantages. An endoscope probe contains laser fibers that allow for effective illumination and viewing, and it is inserted through the nasal pars plana sclerotomy that is created for the vitrectomy [13].

The insertion of the array requires the complete removal of the anterior vitreous in this area to minimize the pulling effect of the residual vitreous remnants; otherwise, the risk of ciliary body detachment and/or a retinal tear will increase. The presence of the residual vitreous tissue can be seen completely with an endoscope because of the normal anatomical contour of the retroiridal tissues and the real-time relationship between the traction forces because a scleral indentation is not performed. This would be an advantage because the electronic case and the receiving coil have already been sutured on the sclera around the globe of the eye.

The creation of a scleral incision for insertion of the array is one of the most important steps of this surgery. Its distance from the limbus is calculated based on the biometric data obtained from the axial length measurement made based on the findings from an ultrasound examination. However, this approach may not be suitable in every case because of developmental variations in the ora serrata such as meridional folds, meridional complexes, and oral bays, which are common and occurred in $47 \%$ of the 204 normal eyes. In moderate or high myopia, the ora serrata is often located posterior to the rectus muscle insertions. The pars plana zone is $3 \mathrm{~mm}$ wide in the nasal quadrants and $5 \mathrm{~mm}$ wide in the temporal quadrants. Fiberoptic transillumination through the pupil can be used to demonstrate ora serrata when choosing the location for the pars plana incision [14]. In eyes with this type of variation in the normal pattern of the pars plana, if a scleral incision is made only based on the axial length measurement, scleral incision-related complications could occur. To minimize these risks, a fine-needle test can be useful.

We controlled the location of the 5.2-mm pars plana incision internally using an endoscope just before making the full thickness incision in order to determine if the incision site was proper. Just after making the scleral and choroidal incisions and inserting the array and cable, it is helpful to see the retroiridal space to determine if any serious complications, such as a retinal tear, ciliary body damage, and/or detachment, have developed.

An important aspect of all epiretinal devices is the distance between the retina's inner surface and the electrodes. To achieve a significant change in the membrane potential in the retina, the stimulating electrodes have to be applied as close to the target cells as possible. Care has to be taken intraoperatively to achieve tight contact between the implant and the retina [15]. With an operating microscope, it is difficult to assess the contact due to the anteroposterior view it provides. Because of the side-viewing capability of an endoscope, a surgeon can assess the conformity of the contact between the array and the inner surface of the macula and the degree of the retinal tack compression. If improper situations are observed, retaking can be considered.

As a general rule, at the end of the vitreoretinal surgery, it is important to check the retroiridal area with the scleral indentation to determine if any serious complications, such as ciliary body/choroidal detachment and retinal tear(s), have developed. Scleral indentation may be limited and harmful since the electronic case and the coil are sutured to the sclera. This can be easily, quickly, and completely accomplished using an endoscope without any damage to the electronic devices. If a retinal tear is detected, it can be treated using an endoscopic endolaser application, and, if necessary, an appropriate intraocular tamponade can be 
chosen. It can also be determined whether there is any tilt or bend in the cable passing through the pars plana incision that could cause a leak or severe hypotony postoperatively. In this case, it is necessary to change the sutures to correct the tilting or bending of the cable.

\section{Conclusion}

Although SAEs and complications occur less frequently due to improvements in the device design and the surgical steps used in Argus II implantation surgery, some sclerotomy incision-related complications can still occur, such as retinal damage, infringement on the ciliary body, inadequate placement of the electrode array over the fovea, and tacking issues. Intraoperative assistance of an ophthalmic microendoscope may further decrease the rate of devastating and severe complications in which treatment might be difficult or unsuccessful. Learning about and using an endoscope is easier in implantation surgery because the natural lens has already been removed. While an endoscope adds an additional cost to the surgery, it is valuable because it prevents the need for difficult additional surgeries and added expense. It also further improves the surgical and functional outcome because control over the surgical procedure is more effective than when using other approaches.

\section{Statement of Ethics}

The study was conducted in accordance with the Declaration of Helsinki and written informed consent was obtained from the patients before reporting their results.

\section{Disclosure Statement}

None of the authors have any proprietary interests or conflicts of interest related to this submission. None of the authors received any financial support.

\section{References}

1 Chuang AT, Margo CE, Greenberg PB: Retinal implants: a systematic review. Br J Ophthalmol 2014;98:852-856.

- Dorn JD, Ahuja AK, Caspi A, et al: The detection of motion by blind subjects with the epiretinal 60-electrode (Argus II) retinal prosthesis. JAMA Ophthalmol 2013;131:183-189.

-3 Yue L, Falabella P, Christopher P, et al: Ten-year follow-up of a blind patient chronically implanted with epiretinal prosthesis Argus I. Ophthalmology 2015;122:2545-2552.e1.

-4 Kasi H, Hasenkamp W, Cosendai G, et al: Simulation of epiretinal prostheses - evaluation of geometrical factors affecting stimulation. J Neuroengineering and Rehabilitation 2011;8:1-10.

5 Ho AC, Humayun MS, Dorn JD, et al: Long-term results from an epiretinal prosthesis to restore sight to the blind. Ophthalmology 2015;122:1547-1554.

-6 Da Cruz L, Coley BF, Dorn J, et al: The Argus II epiretinal prosthesis system allows letter and word reading and long-term function in patients with profound vision loss. Br J Ophthalmol 2013;97:632636.

7 Barry MP, Dagnelie G: Use of the Argus II retinal prosthesis to improve visual guidance of fine hand movements. Invest Ophthalmol Vis Sci 2012;53:5095-5101.

-8 Geruschat DR, Flax M, Tanna N, et al: FLORA ${ }^{\mathrm{TM}}$ : phase I development of a functional vision assessment for prosthetic vision users. Clin Exp Optometry 2015;98:342-347.

9 Hahn P: The next generation of artificial vision. Retina Today 2015;10:61-66.

10 Second Sight Medical Products, Inc.: Clinical update 1, December 2015. Sylmar, CA, USA. 
Özmert and Demirel: Endoscope-Assisted and Controlled Argus II Epiretinal Prosthesis Implantation in Late-Stage Retinitis Pigmentosa: A Report of 2 Cases

11 Walter P (ed): Retina Implant Care - Basics, Procedures and Follow-up. London, Boston, International Medical Publishers, 2014.

12 Second Sight Medical Products, Inc.: Argus II Retinal Prosthesis System surgeon manual. Sylmar, CA, USA.

13 Uram M: Endoscopic Surgery in Ophthalmology. Philadelphia, Baltimore, New York, Lippincott Williams \& Wilkins, 2003.

14 Michelson RG, Wilkinson CP, Rice TA (eds): Anatomy and Physiology. Retinal Detachment. St. Louis, Baltimore, CV Mosby Company, 1990.

15 Tsai D, Chen S, Protti DA, et al: Responses of retinal ganglion cells to extracellular electrical stimulation, from single cell to population: model-based analysis. PLoS One 2012;7:e53357.

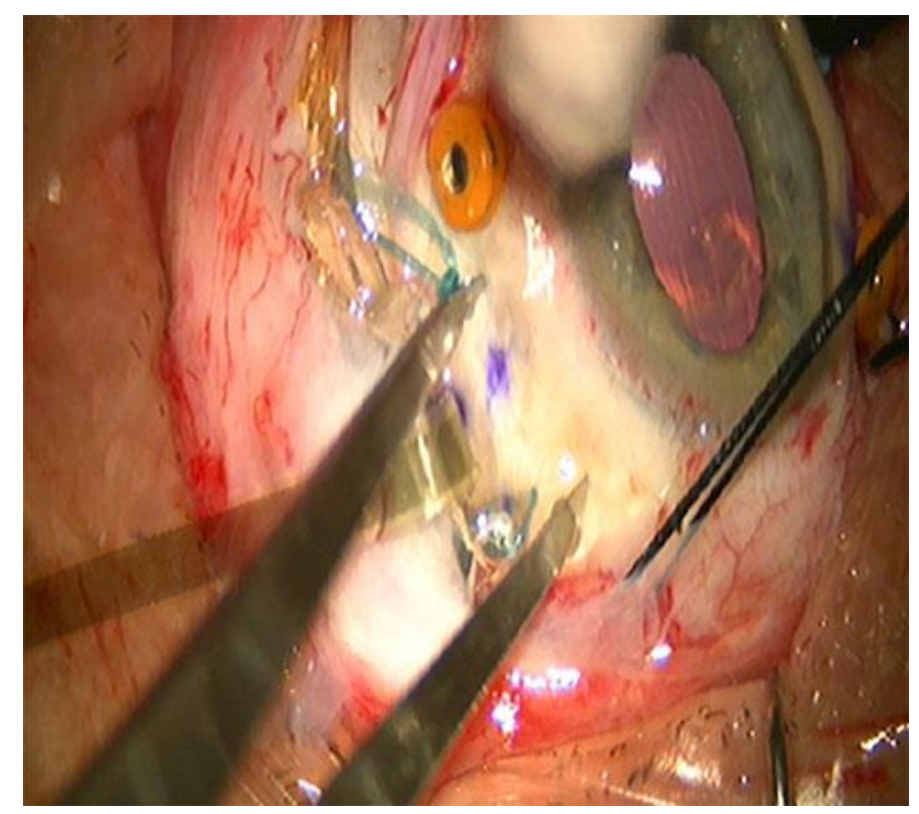

Fig. 1. The prescribed setback distance from the limbus is measured, and the center of the sclerotomy site is marked for the scleral incision. 


\section{Case Reports in Ophthalmology}

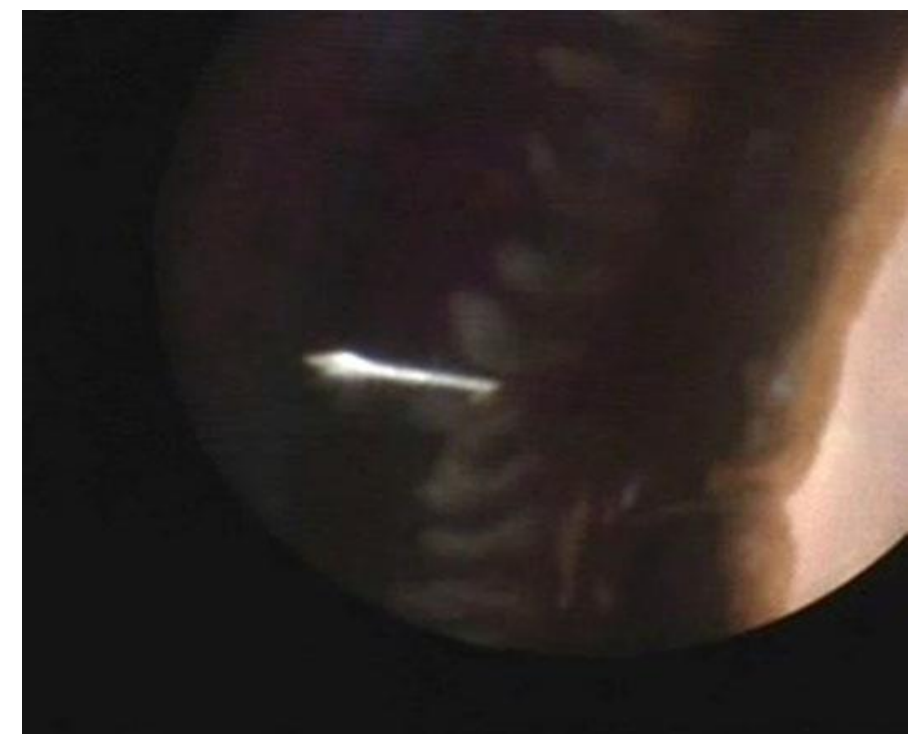

Fig. 2. A 30-G fine needle is inserted through the prepared central mark on the pars plana. The tip of the needle is seen at the border between the ciliary body and the pars plana, using an endoscope.

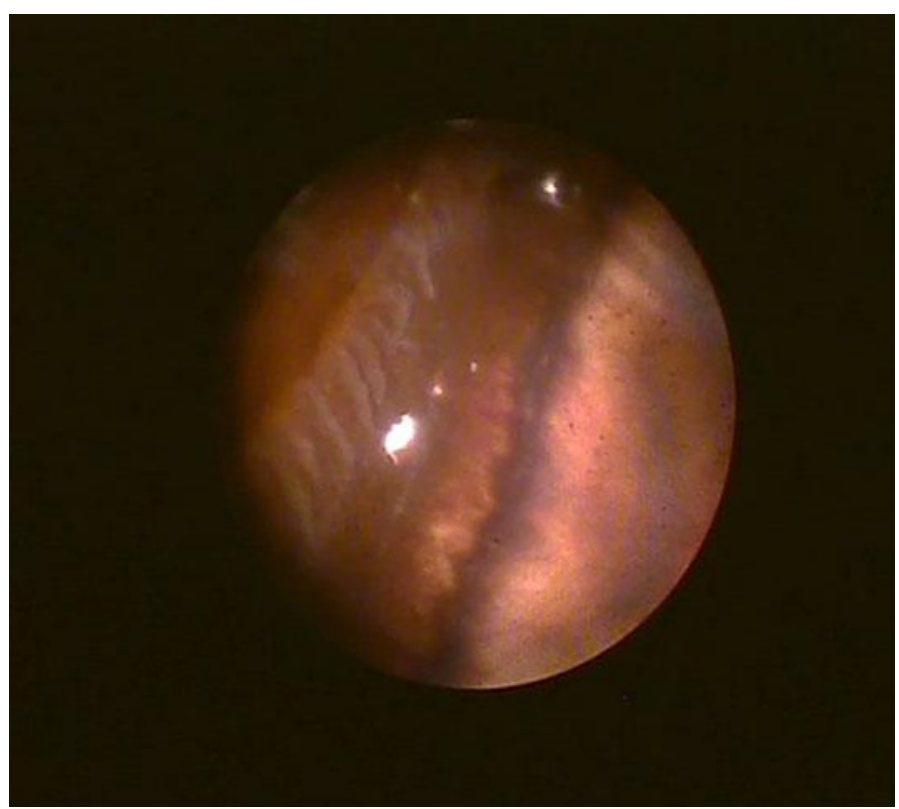

Fig. 3. The pars plana, ora serrata, and the trace of the needle (large white dot) are controlled via direct viewing using an endoscope. The needle trace is located perfectly on the pars plana, indicating that the scleral incision site is suitable. 


\section{Case Reports in Ophthalmology}

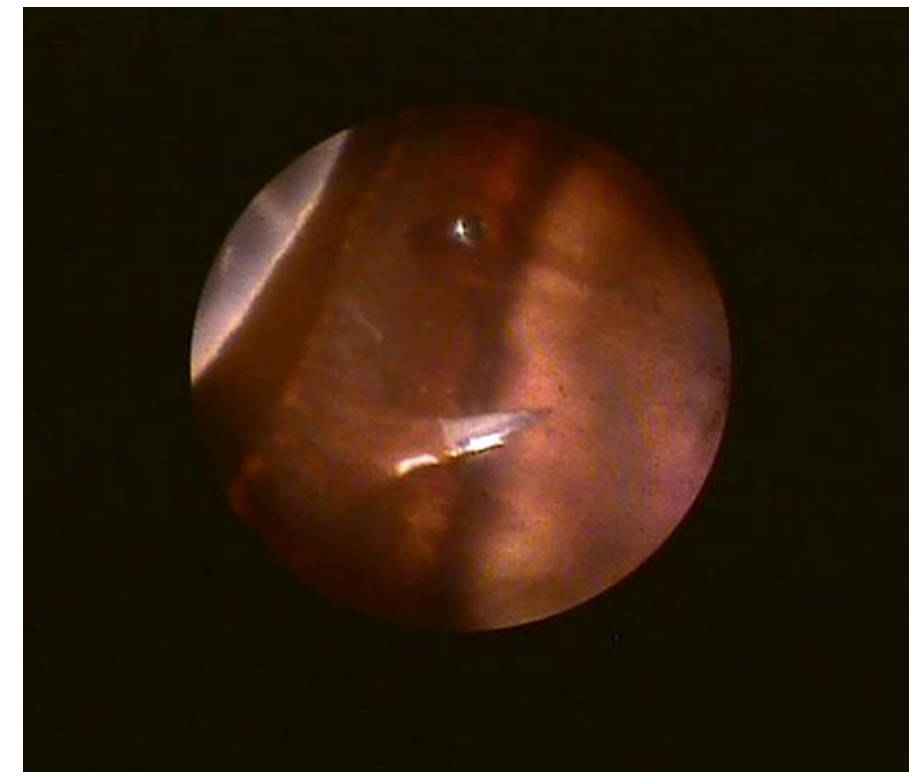

Fig. 4. If a surgeon deems that the scleral incision site is appropriate, 5.2-mm scleral and choroidal incisions are performed using an endoscopic view.

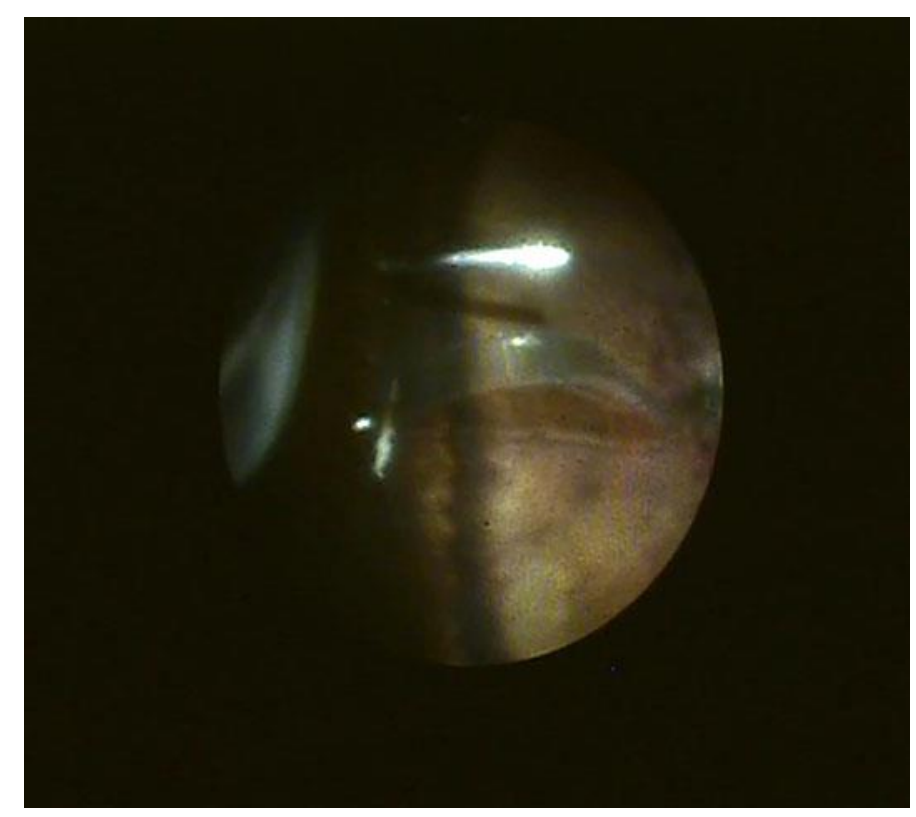

Fig. 5. After insertion of the cable and the array, the retroiridal space is controlled to limit incision- and/or vitreous-related complications, which can be clearly seen with an endoscope without any need for a scleral indentation. An endoscope is also used to determine if there is a tilt or a bend in the electronic cable and improper wound closure, which could lead to severe hypotony postoperatively. 
Case Reports in
Ophthalmology

Case Rep Ophthalmol 2016;7:315-324

\begin{tabular}{l|l}
\hline DOI: $10.1159 / 000453606$ & C 2016 The Author(s). Published by S. Karger AG, Basel
\end{tabular} www.karger.com/cop

Özmert and Demirel: Endoscope-Assisted and Controlled Argus II Epiretinal Prosthesis Implantation in Late-Stage Retinitis Pigmentosa: A Report of 2 Cases

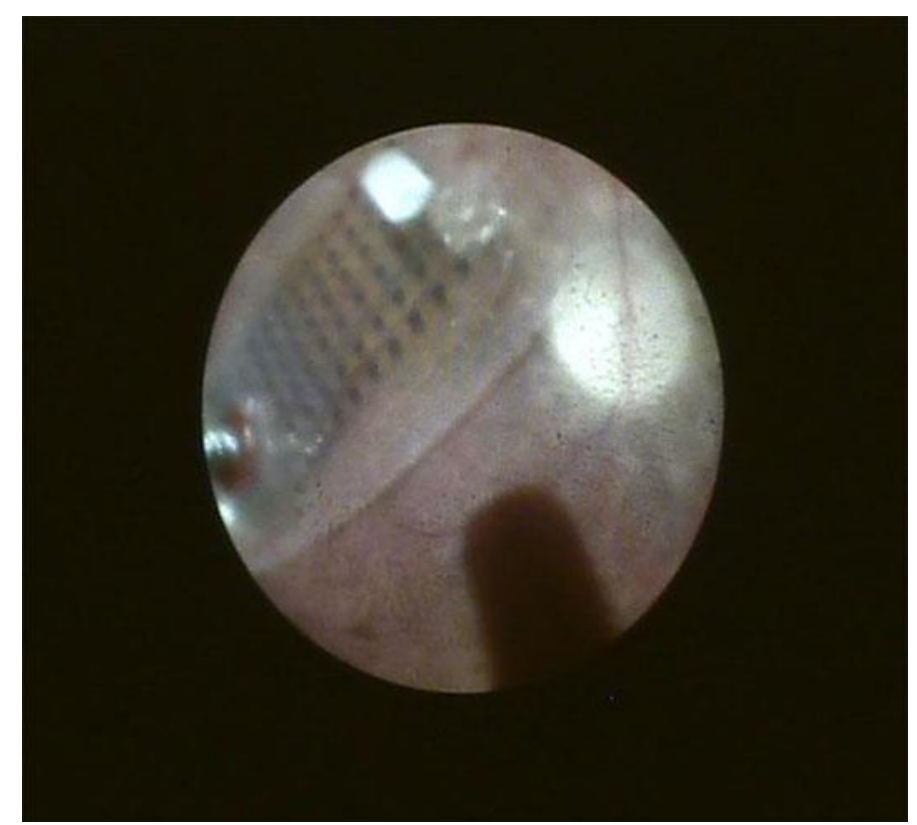

Fig. 6. With the side-viewing capability of a curved endoscope probe, the compression degree of the tack (left), the position of the array in relation to the optic disc, and the contact between the array and the inner surface of the macula can be assessed and clearly determined before ending the surgery. 